\title{
La collaboration enseignant- parent: quelles strategies pour favoriser des relations harmonieuses?
}

\section{Serge J. Larivée et Fernand Ouédraogo}

Depuis les quarante dernières années, de nombreuses recherches ont été réalisées sur les relations école-famille, mais elles portent particulièrement sur la collaboration ou l'implication des parents sans nécessairement traiter en profondeur la dynamique relationnelle entre parents et enseignants. Dans ce texte, nous présentons les résultats d'une recherche qui porte sur les stratégies relationnelles à privilégier pour favoriser l'établissement de relations harmonieuses entre parents et enseignants. Elle a été menée auprès de parents d'enfants $(n=18)$ et d'enseignants $(n=18)$ du préscolaire (maternelle 5 ans) ou du primaire ( $1^{e r}, 2^{e}$ et $3^{e}$ cycle, soit 6 à 11 ans). Nous présentons d'abord le contexte et des obstacles entourant les relations école-famille. Nous exposons ensuite les stratégies qui favorisent des relations parent-enseignant harmonieuses puis nous identifions les perceptions communes et spécifiques qui les caractérisent.

\section{Introduction}

Les relations école-famille se sont profondément transformées avec les années. En effet, alors que les parents demeuraient plutôt à distance de l'école dans les années 1960, ils se sont graduellement davantage impliqués dans la vie scolaire de leur enfant et ils sont aujourd'hui généralement plus présents à l'école ou dans le suivi scolaire de leur enfant. Ce rapprochement entre les parents et l'école, qui est encadré légalement dans plusieurs pays, par exemple dans la Loi sur l'instruction publique dans la province de Québec au Canada, est plus que jamais souhaité et attendu (Dom \& Verhoeven, 2006; Gouvernement du Québec, 2001). Dans les documents gouvernementaux, professionnels ou scientifiques, les parents sont ainsi considérés comme des collaborateurs, voire des partenaires de l'école. Malgré ces changements importants, la collaboration école-famille se vit différemment d'un établissement scolaire ou d'une famille à l'autre, les relations entre les enseignants et les parents demeurant multidimensionnelles, complexes et fragiles. 
La documentation scientifique sur le sujet est assez abondante, mais elle concerne principalement la collaboration ou l'implication des parents sans nécessairement traiter en profondeur la dynamique relationnelle entre les parents et les enseignants. Pour tenter de combler en partie cette lacune, nous avons réalisé une recherche sur les relations parent-enseignant. Dans ce texte, nous présentons une partie des résultats de cette recherche, soit ceux concernant les stratégies relationnelles. Nous débutons d'abord par la présentation du contexte entourant les relations école-famille ainsi que l'identification de divers obstacles rencontrés par les parents et les enseignants. Nous exposons ensuite les résultats de notre recherche sur les stratégies qui favorisent des relations parent-enseignant harmonieuses ${ }^{1}$, telles qu'identifiées par des parents et des enseignants au préscolaire et au primaire. Enfin, nous confrontons leurs réponses respectives afin d'en dégager leurs perceptions communes et celles spécifiques.

\section{Les relations école-famille}

Depuis les quarante dernières années, de nombreuses études ont été menées sur les relations école-famille, particulièrement sur les types d'implication parentale et les effets de la collaboration entre les familles et les instances éducatives intervenant auprès des enfants (Brougère \& Rayna, 2005; Epstein \& Sanders, 2000; Garcia, 2004; Peña, 2000; Rayna, Rubio \& Scheu, 2010). De ces recherches, il apparaît que le rapport qu'entretiennent les parents avec l'école est fort diversifié. Celui-ci varie notamment selon les caractéristiques des parents (niveau de scolarité, statut socioéconomique, occupation,...), l'âge et le parcours scolaire de leur enfant (ex.: s'il a des difficultés). Dès lors, les relations école-famille mettent en évidence deux dimensions particulières mais complémentaires: la collaboration entre le personnel scolaire (principalement les enseignants) et les parents ainsi que l'implication des parents dans le suivi scolaire de leur enfant.

Le Petit Robert de la langue française (Rey, Robert, Rey-Debove \& Cottez, 2006, s.p.) définit la collaboration comme étant un «travail en commun, action de collaborer avec qqn.». Ainsi, le fait de travailler avec quelqu'un à une activité ou à un projet commun constitue une forme de collaboration. Le Petit Robert de la langue française montre que le terme "collaboration" est générique; il fait référence à d'autres termes apparentés tels l'aide, l'appui, le concours, la coopération, la participation. C'est ce qui explique pourquoi la documentation scientifique n'en présente pas une définition stable, commune ou partagée.

C'est toutefois plus clair en contexte scolaire où la collaboration école-famille-communauté est le fait pour les différents acteurs concernés (parents, personnels scolaires, membres de la communauté) de travailler ensemble dans un but commun, soit contribuer, directement ou indirectement, à la réussite éducative ou scolaire de l'élève. Elle vise donc, implicitement, à ce que les différents acteurs s'impliquent selon leurs rôles respectifs. En outre, on peut retenir 
que la collaboration repose sur les relations entre les acteurs alors que l'implication constitue les actions qu'ils posent pour favoriser la réussite éducative ou scolaire de l'élève.

Ces deux dimensions (collaboration et implication) sont reconnues dans la documentation scientifique comme étant des facteurs d'influence importants de la réussite scolaire (Caspe, Lopez \& Wolos, 2007; Deslandes \& Bertrand, 2004). En effet, les recherches menées sur la collaboration école-famille-communauté (ÉFC) ou, plus spécifiquement, sur l'implication parentale dans le suivi scolaire ont mis en évidence les effets positifs que peuvent avoir les parents dans le développement et la réussite scolaire de leur enfant, et ce, peu importe l'âge ou le niveau scolaire (préscolaire, primaire, secondaire), le milieu socioéconomique (favorisé, moyen, favorisé) ou les caractéristiques personnelles de l'élève (Epstein, 2001, 2008; Henderson \& Mapp, 2002; Jeynes, 2011; Larivée \& Larose, 2014). L'identification de stratégies pouvant favoriser l'établissement et l'amélioration des relations harmonieuses entre les enseignants et les parents apparait comme l'une des pistes pouvant contribuer à améliorer la collaboration et l'implication des parents et, indirectement, contrer l'échec scolaire et favoriser la réussite d'un plus grand nombre d'élèves, particulièrement ceux étant les plus vulnérables. Cependant, malgré la bonne volonté d'un grand nombre de parents et d'enseignants, il semble que l'établissement de relations harmonieuses reposant sur une véritable collaboration parent-enseignant soit un défi permanent en raison d'obstacles multiples.

\section{Des obstacles aux relations école-famille}

Comme tout type de relations interpersonnelles, celles impliquant les enseignants et les parents sont parsemées d'obstacles qui influencent leur qualité. Il importe donc d'identifier ces obstacles afin de les apprivoiser et de tenter de les contrer par diverses stratégies. À ce sujet, le modèle du processus de participation parentale de Hoover-Dempsey et Sandler (1997) identifient des obstacles qui peuvent expliquer pourquoi les parents ne collaborent ou ne s'impliquent pas ou peu. Ils citent, par exemple, les responsabilités familiales ou professionnelles, le faible niveau d'éducation des parents, les représentations erronées quant aux rôles parentaux, le manque de clarté dans les exigences ou les attentes de l'école à leur égard ou à l'égard de leur enfant, la valeur accordée à l'éducation par les parents, etc.

Un autre obstacle concerne le statut de chaque catégorie d'acteurs et le pouvoir que ce statut confere. D’entrée de jeu, cela crée un déséquilibre dans la relation qui peut être accentué selon la façon dont chacun interprète et joue son rôle. En effet, les enseignants détiennent une expertise qui leur donne un pouvoir sur les apprentissages et la réussite scolaires de l'enfant. Dès lors, ils ont un minimum d'obligations et de responsabilités tant envers l'enfant qu'envers les parents. De 
leur côté, les parents sont responsables de l'éducation de leur enfant. Ils ont notamment l'obligation et la responsabilité de répondre aux besoins de base de l'enfant et de veiller à ce qu'il fréquente l'école dans les meilleures conditions possible. Il est aussi attendu qu'ils collaborent avec l'école et qu'ils s'impliquent dans le suivi scolaire de l'enfant. Or, les parents n'accordent pas la même importance et la même confiance envers l'école ou les enseignants et il en est de même pour les enseignants à l'égard des parents, ce qui influence leurs relations et leur collaboration. Ainsi, comment juger de cette collaboration ou implication? Qu'est-ce qui est acceptable ? Il existe un flou sur ce qui est attendu de part et d'autre, ce qui varie d'un parent à l'autre ou d'un enseignant à l'autre, et qui contribue à maintenir une ambivalence relationnelle entre ces deux catégories d'acteurs hétérogènes (Crozier, 2000).

Un autre obstacle concerne la dimension temporelle. Tant les parents que les enseignants mentionnent qu'ils manquent de temps (Deniger, Anne, Dubé \& Goulet, 2009; Larivée, 2012). Les enseignants estiment ne pas avoir le temps nécessaire pour communiquer plus souvent avec les parents ou pour organiser des activités de collaboration ou d'implication parentale. De leur côté, les parents affirment manquer de temps pour communiquer ou rencontrer les enseignants et s'impliquer à l'école. Ce manque de temps influence ainsi le type de collaboration enseignant-parent et, par le fait même, la qualité de la relation. Celle-ci sera toutefois influencée, positivement ou négativement, selon les perceptions et attentes respectives quant aux rôles de chacun. Ainsi, un enseignant et un parent qui ont des perceptions similaires de leurs rôles respectifs ont plus de chance d'être sur la même longueur d'onde et d'établir des relations harmonieuses que l'inverse.

La peur du jugement de l'autre est aussi un obstacle important. De nombreux parents ont peur, par exemple, d'être incompris ou jugés incompétents par le personnel scolaire (Ouédraogo, 2016) et il en est de même pour les enseignants à l'égard des parents, particulièrement dans un contexte d'implication des parents en classe. Cette crainte du jugement se traduit parfois par l'adoption d'une position défensive menant à faire porter le blâme sur l'autre, et ce, tant de la part des parents que des enseignants ou chacun attribue la responsabilité des difficultés et des échecs de l'élève à l'autre (Claes \& Comeau, 1996; Letarte, Nadeau, Lessard, Normandeau \& Allard, 2011). Ce type de comportement mine considérablement la relation qui devient, à la longue, difficile à entretenir et à améliorer.

D'autres obstacles de nature et d'importance variées influencent les relations école-famille et, plus particulièrement, les types de collaboration et d'implication parentale. A cet égard, Larivée (2011) identifie quatre catégories de facteurs d'influence, soit ceux d'ordre: 1) organisationnel (structure familiale, conciliation travail-famille-école, etc.); 2) relationnel (accueil et attitudes des enseignants, etc.); 3) psychologique (représentation du rôle de l'école, vécu scolaire, etc.); 4) individuel (âge des enfants, présence de difficultés scolaires, 
etc.). Il apparaît aussi que les obstacles qui nuisent aux relations entre parents et enseignants reposent particulièrement sur des malentendus, des incompréhensions, des contradictions et des revendications mutuelles (Dubet, 1997; Périer, 2005) dont les origines sont habituellement liées au rendement scolaire ou aux comportements de l'enfant.

En outre, au regard des éléments présentés ci-dessus, il apparaît que les relations famille-école, ou plus spécifiquement parent-enseignant, sont complexes et multidimensionnelles. Il faut garder en tête que les parents et les enseignants sont des entités hétérogènes, ce qui complexifie davantage la nature de leurs relations. Bien que de nombreux praticiens souhaiteraient connaître la manière d'interagir avec les parents (ou la recette) pour établir et maintenir des relations harmonieuses, force est de constater qu'il n'y a pas une seule façon d'agir pour garantir ce succès (Ouédraogo, 2016). Cependant, des stratégies favorisant de telles relations peuvent sûrement être identifiées. Dès lors, deux questions de recherche ont émergé à ce sujet: 1) quelles sont les stratégies favorisant des relations harmonieuses entre parents et enseignants? 2) quels sont les perceptions communes et spécifiques qui les caractérisent?

\section{Les objectifs de recherche}

Ces deux questions de recherche ont été abordées dans le cadre d'une recherche plus large dont l'objectif général est d'élaborer et de valider un modèle théorique reposant sur les rôles éducatifs et le profil (types) des parents et des enseignants ainsi que sur les types d'implication parentale favorisant la réussite scolaire des enfants au préscolaire ou au primaire (Larivée, 2010-2012).

Dans cet article, nous porterons cependant notre attention sur les deux objectifs spécifiques suivants:

1) identifier les perceptions de parents à l'égard des stratégies favorisant l'établissement de relations harmonieuses entre les parents et les enseignants;

2) identifier les perceptions d'enseignants à l'égard des stratégies favorisant l'établissement de relations harmonieuses entre les parents et les enseignants.

\section{Mé thodologie}

\section{Caractéristiques des participants}

Pour atteindre les objectifs de cette recherche, nous souhaitions interroger des parents et des enseignants puisque, comme le souligne Beauregard (2006), «la façon dont un parent ou un enseignant conçoit son rôle auprès de l'enfant ou de l'élève s'appuie sur sa compréhension et sa vision de la situation dans laquelle il exerce son rôle» (p. 547). Nous avons ainsi tenté de former six groupes de discussion, soit trois constitués de parents d'enfants du préscolaire (maternelle 5 
ans) et du primaire ( $1^{\mathrm{er}}, 2^{\mathrm{e}}$ et $3^{\mathrm{e}}$ cycles) et trois d'enseignants au préscolaire et au primaire. L'échantillon de parents et celui d'enseignants devaient être composés de 18-24 participants chacun (trois groupes de 6-8 parents, trois groupes de 6-8 enseignants), issus de régions (milieux rural, suburbain et urbain) et de milieux socioéconomiques (défavorisés, moyens et favorisés) différents. Ces critères visaient à favoriser une diversité et une complémentarité des points de vue. Les participants ont été recrutés sur une base de volontariat par le biais des commissions scolaires selon l'indice socioéconomique du ministère de l'éducation, du Loisir et du Sport (MÉLS), soit les cotes 1 et 2 pour les écoles de milieux favorisés, 5 et 6 pour celles des milieux moyens et 9 et 10 pour celles des milieux défavorisés. Cependant, notamment en raison de moyens de pression exercés par les enseignants dans le cadre des négociations de leur convention collective, il a été impossible de procéder comme nous l'avions envisagé. De plus, étant donné que les participants ayant accepté de participé ne résidaient pas nécessairement dans des environnements rapprochés, il ne nous a pas été possible de former trois groupes de parents et trois groupes d'enseignants. Nous avons donc plutôt réalisé des entrevues individuelles ou de groupe (2-3 personnes). L'échantillon réel est donc composé de 18 parents d'enfants du préscolaire (maternelle 5 ans) et du primaire ( $1^{\mathrm{er}}, 2^{\mathrm{e}}$ et $3^{\mathrm{e}}$ cycles) ainsi que de 18 enseignants au préscolaire et au primaire. Ceux-ci ne sont pas liés entre eux, c'est-à-dire que les enfants des parents participants ne fréquentaient pas les classes de ces enseignants. Les entrevues se sont déroulées comme suit: 15 entrevues individuelles et 3 entrevues de groupe de 2 personnes pour les parents ; 5 entrevues individuelles et 5 entrevues de groupe ( 2 groupes de 2 personnes et 3 groupes de 3 personnes) pour les enseignants.

Pour ce qui concerne les enseignants, ceux-ci sont issus de six commissions scolaires, soit quatre de l'île de Montréal, treize de la Rive-Nord et une de la Rive-Sud de Montréal. Les enseignants (cf. tableau 1), presque entièrement des femmes (94\%) nés au Québec (94\%), sont majoritairement âgés entre 26 et 45 ans $(61 \%)$. Plus de $78 \%$ d'entre eux détiennent un baccalauréat en éducation préscolaire et enseignement primaire et ont une expérience professionnelle de plus de 11 ans. La majorité occupe un poste permanent $(67 \%)$, enseigne à temps plein $(89 \%)$ et intervient à l'ordre primaire $(67 \%)$. Enfin, $72 \%$ des enseignants affirment s'impliquer dans des activités à l'école autres que l'enseignement (membre du conseil d'établissement, comité de travail, etc.). 
Tableau 1. Caractéristiques des enseignants

\begin{tabular}{|c|c|c|}
\hline Caractéristiques & Nombre & $\%$ \\
\hline $\begin{array}{ll}\text { Sexe } & \\
\text { - } & \text { Féminin } \\
\text { - } & \text { Masculin }\end{array}$ & $\begin{array}{c}17 \\
1\end{array}$ & $\begin{array}{c}94,4 \\
5,6\end{array}$ \\
\hline $\begin{aligned} \text { Âge } & \\
- & 25 \text { ans } \\
- & 26-30 \text { ans } \\
- & 31-35 \text { ans } \\
- & 36-40 \text { ans } \\
- & 41-45 \text { ans } \\
- & 46-50 \text { ans } \\
- & 51 \text { et }+\end{aligned}$ & $\begin{array}{l}0 \\
5 \\
0 \\
3 \\
6 \\
2 \\
1\end{array}$ & $\begin{array}{c}0,0 \\
29,4 \\
0,0 \\
17,6 \\
35,3 \\
11,8 \\
5,9\end{array}$ \\
\hline 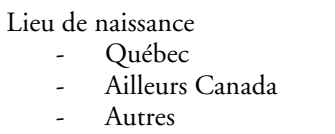 & $\begin{array}{c}17 \\
0 \\
1\end{array}$ & $\begin{array}{c}94,4 \\
0,0 \\
5,6\end{array}$ \\
\hline $\begin{aligned} \text { Scolarité } & \\
- & \text { BEPEP } \\
- & \text { BEAS } \\
- & \text { Autres }\end{aligned}$ & $\begin{array}{c}14 \\
4 \\
0\end{array}$ & $\begin{array}{c}77,8 \\
22,4 \\
0,0\end{array}$ \\
\hline $\begin{array}{l}\text { Expérience en enseignement } \\
\begin{aligned}- & -5 \text { ans } \\
- & 6-10 \text { ans } \\
- & 11-20 \text { ans } \\
- & + \text { de } 20 \text { ans }\end{aligned}\end{array}$ & $\begin{array}{l}4 \\
2 \\
8 \\
4\end{array}$ & $\begin{array}{l}22,2 \\
11,1 \\
44,4 \\
22,2\end{array}$ \\
\hline $\begin{array}{l}\text { Statut du poste } \\
-\quad \text { Permanent } \\
-\quad \text { Non permanent }\end{array}$ & $\begin{array}{c}12 \\
6\end{array}$ & $\begin{array}{l}66,7 \\
33,3\end{array}$ \\
\hline 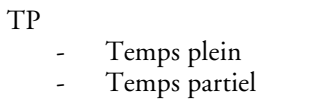 & $\begin{array}{c}16 \\
2\end{array}$ & $\begin{array}{l}88,8 \\
11,2\end{array}$ \\
\hline $\begin{aligned} \text { Ordre d'enseignement } \\
-\quad \text { Préscolaire } \\
-\quad \text { Primaire } 1^{\text {er }} \text { cycle } \\
-\quad \text { Primaire } 2^{\mathrm{e}} \text { cycle } \\
-\quad \text { Primaire } 3^{\mathrm{e}} \text { cycle } \\
-\quad \text { Divers }\end{aligned}$ & $\begin{array}{l}6 \\
4 \\
4 \\
3 \\
1\end{array}$ & $\begin{array}{r}33,3 \\
22,2 \\
22,2 \\
16,7 \\
5,6\end{array}$ \\
\hline $\begin{array}{c}\text { Implication à l'école } \\
-\quad \text { Oui } \\
-\quad \text { Non }\end{array}$ & $\begin{array}{c}13 \\
5\end{array}$ & $\begin{array}{l}72,2 \\
27,8\end{array}$ \\
\hline
\end{tabular}

Pour ce qui concerne les parents, deux habitent sur le territoire scolaire de l'île de Montréal, trois sur la Rive-Sud et 13 sur la Rive-Nord de Montréal. Les parents de l'échantillon (cf. tableau 2), sont principalement des mères (72\%), nés au Québec (61\%), âgés de 31 ans ou plus (83\%) et mariés ou vivant en union libre (78 \%). À propos du profil familial, $61 \%$ des mères ont un diplôme universitaire contre $28 \%$ des pères. Près des trois quarts $(72 \%)$ des parents occupent un 
emploi régulier rémunéré pour lequel $61 \%$ gagne un revenu familial supérieur à 75000 \$. Enfin, la majorité des parents $(61 \%)$ ont un ou deux enfants et affirment ne pas s'impliquer dans des activités à l'école (membre du conseil d'établissement, comité de travail, etc.).

Tableau 2. Caractéristiques des parents

\begin{tabular}{|c|c|c|c|c|}
\hline Caractéristiques & \multicolumn{2}{|c|}{ Nombre } & \multicolumn{2}{|c|}{$\%$} \\
\hline $\begin{array}{cl}\text { Statut du parent participant } \\
\text { - } & \text { Père } \\
\text { - } & \text { Mère } \\
\text { - } & \text { Beau-père } \\
\text { - } & \text { Belle-mère } \\
\text { - } & \text { Autre }\end{array}$ & & & & \\
\hline $\begin{aligned} & \text { Statut familial } \\
&- \text { Marié ou en union libre } \\
&- \text { Monoparental } \\
&- \text { Non répondu }\end{aligned}$ & \multicolumn{2}{|c|}{$\begin{array}{c}14 \\
2 \\
2\end{array}$} & \multicolumn{2}{|c|}{$\begin{array}{l}77,8 \\
11,1 \\
11,1\end{array}$} \\
\hline 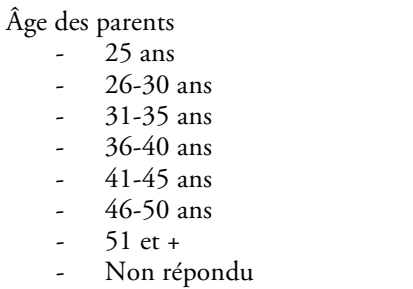 & $\begin{array}{l}\text { Mère } \\
0 \\
0 \\
5 \\
4 \\
5 \\
1 \\
0 \\
3\end{array}$ & $\begin{array}{l}\text { Père } \\
1 \\
0 \\
3 \\
3 \\
3 \\
2 \\
2 \\
4\end{array}$ & $\begin{array}{l}\text { Mère } \\
0,0 \\
0,0 \\
27,8 \\
22,2 \\
27,8 \\
5,6 \\
0,0 \\
16,7\end{array}$ & $\begin{array}{c}\text { Père } \\
5,6 \\
0,0 \\
16,7 \\
16,7 \\
16,7 \\
11,1 \\
11,1 \\
22,2\end{array}$ \\
\hline $\begin{array}{c}\text { Lieu de naissance des parents } \\
\text { - } \text { Québec } \\
\text { - } \\
\text { - } \\
\text { - } \\
\text { Autleurs Canada } \\
\text { Non répondu }\end{array}$ & $\begin{array}{l}\text { Mère } \\
11 \\
0 \\
5 \\
2\end{array}$ & $\begin{array}{l}\text { Père } \\
10 \\
0 \\
4 \\
4\end{array}$ & $\begin{array}{l}\text { Mère } \\
61,1 \\
0,0 \\
27,8 \\
11,1\end{array}$ & $\begin{array}{l}\text { Père } \\
55,6 \\
0,0 \\
22,2 \\
22,2\end{array}$ \\
\hline $\begin{array}{cl}\text { Scolarité des parents } \\
- & \text { Primaire } \\
- & \text { Secondaire } \\
- & \text { Collégial } \\
- & \text { Universitaire } \\
- & \text { Non répondu }\end{array}$ & $\begin{array}{l}\text { Mère } \\
0 \\
0 \\
4 \\
11 \\
3\end{array}$ & $\begin{array}{l}\text { Père } \\
1 \\
3 \\
4 \\
5 \\
5\end{array}$ & $\begin{array}{l}\text { Mère } \\
0,0 \\
0,0 \\
22,2 \\
61,1 \\
16,7\end{array}$ & $\begin{array}{r}\text { Père } \\
5,6 \\
16,7 \\
22,2 \\
27,8 \\
27,8\end{array}$ \\
\hline $\begin{array}{l}\text { Statut d'emploi des parents } \\
-\quad \text { A un emploi rémunéré } \\
\text { - N'a pas d'emploi rémunéré } \\
\text { - }\end{array}$ & $\begin{array}{l}\text { Mère } \\
13 \\
2 \\
3\end{array}$ & $\begin{array}{l}\text { Père } \\
13 \\
0 \\
5\end{array}$ & $\begin{array}{l}\text { Mère } \\
72,2 \\
11,1 \\
16,7\end{array}$ & $\begin{array}{l}\text { Père } \\
72,2 \\
0,0 \\
27,8\end{array}$ \\
\hline $\begin{array}{cl}\text { Revenu familial brut } \\
\text { - } & \text { - de } 24999 \$ \\
- & 25000 \$ \text { à } 49999 \$ \\
- & 50000 \$ \text { à } 74999 \$ \\
- & 75000 \text { et + } \\
\text { - } & \text { Non répondu }\end{array}$ & & & & \\
\hline
\end{tabular}




\begin{tabular}{|c|c|c|}
\hline Nombre d'enfants & & \\
$-\quad 1$ ou 2 & 11 & 61,1 \\
$-\quad 3$ ou 4 & 6 & 33,3 \\
- de 4 & 0 & 0,0 \\
- Non répondu & 1 & 5,6 \\
\hline Implication à l'école & 6 & \\
- Oui & 11 & 33,3 \\
- Non & 1 & 61,1 \\
- Non répondu & & 5,6 \\
\hline
\end{tabular}

L'instrumentation: Le protocole d'animation des groupes de discussion

Le protocole d'animation des GD/entrevues visait à recueillir les perceptions des participants sur les thèmes suivants: les rôles éducatifs des parents et des enseignants; les relations parents-enseignants (caractéristiques de parents et d'enseignants, continuité/discontinuité des attentes, stratégies relationnelles); les types de participation parentale dans le suivi scolaire de l'enfant; les conditions favorisant la réussite scolaire. Afin de favoriser la réflexion ainsi que les échanges entre les divers participants des GD, les consignes et les thèmes traités lors des discussions ont été transmis aux participants environ une semaine avant le début des rencontres afin de leur permettre de s'y préparer (Reason, 1994). Toutes les discussions ont été enregistrées (audio) avec l'accord des participants, ce qui a facilité la retranscription et l'analyse des données. Dans ce texte, seuls les résultats en lien avec la question suivante sont présentés, soit la seule qui portait spécifiquement sur les stratégies relationnelles:

Q.11 Pouvez-vous identifier des stratégies pour favoriser de bonnes relations entre les parents et les enseignants? a) Comment les parents peuvent-ils favoriser de bonnes relations avec les enseignants?, b) Comment les enseignants peuvent-ils favoriser de bonnes relations avec les parents?

\section{Présentation des résultats}

À la suite des GD, nous avons retranscrit les verbatims puis procédé à une analyse de contenu thématique (Bardin, 2013) à l'aide du logiciel QDA Miner. De cette analyse, quatre catégories de stratégies relationnelles ont été identifiées, soit celles portant principalement sur: l'implication des parents (I); les apprentissages/le développement de l'enfant/élève (A); les attitudes ou comportements prosociaux (ACP); d'autres aspects d'influence (AA). L'analyse a aussi tenu compte de la ou les personne(s) ciblée(s) par les stratégies nommées (enseignants, parents, enseignants et parents) en plus de mettre en évidence les points communs et spécifiques dans les perceptions des parents et des enseignants (Cf. tableaux 3-4-5). 


\section{Tableau 3. Stratégies identifiées par les enseignants et les parents}

Stratégies concernant les enseignants et les parents

- Attitude d'ouverture, de respect, de confiance mutuelle. (ACP)

- Communication efficace (agenda, téléphone, courriel...). (ACP)

- Ne pas laisser les situations se détériorer ou dégénérer (prévenir les difficultés). (ACP)

\section{Stratégies concernant spécifiquement les enseignants}

- Être accueillant. (ACP)

- Inviter les parents à venir en classe ou à l'école, notamment pour un motif positif (visite, spectacle, dîner communautaire, rencontre de bulletin, journal...). (I)

- Rendre l'école plus accessible aux parents (facilité de voir les enseignants et l'administration, faire de la publicité). (I)

Stratégies concernant spécifiquement les parents

- $\quad \mathrm{Nil}$

\section{Tableau 4. Stratégies identifiées seulement par les parents}

\section{Stratégies concernant les enseignants et les parents}

- Communication constructive. (ACP)

- Rester humble et diplomate, aborder les sujets avec tact. (ACP)

- Avoir des buts et des objectifs communs. (I)

- Travailler en équipe. (I)

\section{Stratégies concernant spécifiquement les enseignants}

- Faire bonne impression lors des premières rencontres avec les parents. (ACP)

- Favoriser la collaboration (participation) dès les premières rencontres avec les parents. (I)

\section{Stratégies concernant spécifiquement les parents}

- Encouragement des enseignants (mot de félicitation, cadeaux...). (ACP)

- Que les parents évitent de déstabiliser les enseignants. (ACP)

\section{Tableau 5. Stratégies identifiées seulement par les enseignants}

Stratégies concernant les enseignants et les parents

- Attitude d'empathie, de loyauté, d'honnêteté, de flexibilité. (ACP)

- Éviter les jugements gratuits, ne pas se fier aux apparences, ne pas dénigrer. (ACP)

- S'expliquer lorsqu'il y a des désaccords pour éviter les malentendus ou les incompréhensions. (ACP)

- Ne pas mettre d'étiquettes aux élèves, parents et enseignants. (ACP)

- Prendre du recul, être capable d'introspection, dédramatiser les situations. (ACP)

- Comprendre les messages implicites. (ACP)

- Admettre ses erreurs et accepter les erreurs des autres. $(\mathrm{ACP}) \bullet \quad$ Faire preuve de persévérance. (ACP)

- $\quad$ Être capable de compromis. (ACP)

- Être capable d'humour. (ACP)

- Éviter les rapports de force, les jeux de pouvoir. (ACP)

- Prendre des décisions pour le bien de l'élève (ses apprentissages, ses comportements, son développement). (A)

- Accepter de travailler avec les autres personnes en charge de l'éducation de l'élève. (A)

- S'informer mutuellement de ce qui se passe à l'école et à la maison, du cheminement de l'élève. (A)

- Clarifier et respecter les rôles de chacun (parents et enseignants), préciser ses attentes. (I) 
- Se rencontrer (à la demande de l'enseignant ou du parent) informellement ou formellement lorsque nécessaire (pour parler de l'élève). (I)

\begin{tabular}{|ll|}
\hline \multicolumn{1}{|c|}{ Stratégies concernant spécifiquement les enseignants } \\
\hline - & S'informer des possibilités (ou disponibilités) d'implication des parents. (I) \\
- & Tenter d'impliquer les deux parents (inviter les parents, expliquer ce qu'ils peuvent faire). (I) \\
- & Sécuriser, rassurer les parents et l'enfant. (I) \\
- & Conscientiser le parent à son rôle éducatif. (I) \\
- & Laisser des choix aux parents. (I) \\
- & Se mettre à la place du parent afin d'être clair, simple et efficace. (I) \\
- & Aider, soutenir les parents dans leur tâche éducative à la maison (la supervision des devoirs et \\
- & leçons). (I) \\
- & Ampliquer le fonctionnement la formation initiale et continue des enseignants sur les relations école-famille. (AA) \\
- & Décrocher et prendre du temps pour soi. (AA) \\
\hline & $\quad$ Stratégies concernant spécifiquement les parents \\
\hline - & Nil \\
\hline
\end{tabular}

\section{Les perceptions communes sur les stratégies relationnelles}

Parmi les stratégies relationnelles identifiées par les enseignants et les parents, quelques-unes peuvent être qualifiées de stratégies générales parce qu'elles concernent ces deux catégories d'acteurs. Elles ciblent des attitudes ou comportements prosociaux à adopter par les enseignants et les parents, tels l'établissement d'une communication efficace (via l'agenda, le téléphone, le courriel...) ainsi que l'ouverture, le respect et la confiance mutuelle (Epstein, 2001, 2008). Les enseignants et les parents soulignent aussi la nécessité de prévenir les difficultés en ne laissant pas les situations se détériorer ou dégénérer.

Les enseignants et les parents ont également identifié quelques stratégies complémentaires qui concernent spécifiquement les enseignants. Ils soulignent que le fait d'être accueillants et d'inviter les parents à venir en classe ou à l'école pour un motif positif (un spectacle, un dîner communautaire, une exposition, etc.) sont des moyens pour améliorer les relations ainsi que la présence des parents à l'école (Hoover-Dempsey \& Sandler, 1997). Selon eux, il importe de rendre l'école plus accessible aux parents, notamment en facilitant les échanges avec les enseignants ou l'administration scolaire, ce qui pourrait inciter les parents à s'impliquer à l'école. Les enseignants et les parents mettent ici en relation un comportement prosocial (être accueillant) pour favoriser l'implication parentale.

Pour ce qui concerne des stratégies spécifiques aux parents, ni les enseignants ni les parents n'en ont identifiées.

\section{Les perceptions spécifiques sur les stratégies relationnelles}

Outre les stratégies relationnelles communes, les parents et les enseignants en ont identifié plusieurs qui étaient différentes par leur nature ou la catégorie de 
personnes à qui elles s'adressent. Comme pour les stratégies communes, nous présentons d'abord les stratégies générales qui concernent tant les enseignants que les parents puis les stratégies spécifiques aux enseignants ou aux parents.

\section{Les stratégies identifiées spécifiquement par les parents}

D’une manière générale, les stratégies identifiées par les parents mettent en évidence l'implication parentale et les comportements prosociaux. En ce qui concerne les stratégies s'adressant aux parents et aux enseignants, les parents mentionnent l'importance d'une communication constructive, d'avoir des buts et des objectifs communs et de travailler en équipe (parents-enseignants). Plus spécifiquement, ils soulignent le fait, tant pour les parents que les enseignants, de rester humbles et diplomates, d'aborder les sujets avec tact.

Pour ce qui concerne spécifiquement les enseignants, les parents indiquent le fait pour ceux-ci de faire bonne impression et de favoriser la collaboration dès les premières rencontres avec les parents. Puis, concernant spécifiquement les parents, ils soulignent l'importance d'encourager les enseignants dans leur travail.

\section{Les stratégies identifiées spécifiquement par les enseignants}

Pour leur part, les enseignants ont identifié un nombre plus élevé de stratégies que les parents. Tout comme les parents, ces stratégies concernent principalement l'implication parentale et les comportements prosociaux. Toutefois, les enseignants identifient aussi quelques stratégies qui se rapportent aux apprentissages de l'élève. Deux autres stratégies s'intéressent à l'enseignant au plan professionnel ou personnel (l'amélioration de la formation et le fait de prendre du temps pour soi-même).

En ce qui a trait aux stratégies générales concernant les parents et les enseignants telles que rapportées par les enseignants, elles sont de l'ordre des attitudes et des comportements prosociaux: faire preuve d'empathie, de loyauté, d'honnêteté, de flexibilité, d'humour et de persévérance; éviter les rapports de force, les jeux de pouvoir, les jugements gratuits, les étiquettes; être capable d'introspection, de s'expliquer lorsqu'il y a des désaccords pour éviter les malentendus ou les incompréhensions, de dédramatiser les situations, d'admettre ses erreurs et d'accepter les erreurs des autres; comprendre les messages implicites. Pour favoriser l'implication parentale, les enseignants soulignent l'importance de clarifier et de respecter les rôles de chacun (parents et enseignants), de préciser ses attentes et de se rencontrer informellement ou formellement lorsque nécessaire, que ce soit à la demande de l'enseignant ou du parent. À propos des stratégies pour favoriser les apprentissages des élèves, il est mentionné l'intérêt de s'informer mutuellement (parents et enseignants) de ce qui se passe à l'école et à la maison, et ce, afin de prendre des décisions pour le bien de l'élève. Enfin, il apparait important pour les enseignants que toutes les personnes en charge de l'éducation travaillent ensemble. 
En ce qui concerne spécifiquement les enseignants, les stratégies identifiées par ces derniers concernent presque exclusivement l'implication des parents à la maison ou à l'école. Il s'agit: de sécuriser et de rassurer les parents et leur enfant, de conscientiser le parent à son rôle éducatif, d'aider ou de soutenir le parent dans leur tâche éducative à la maison (ex.: supervision des devoirs et des leçons), d'expliquer le fonctionnement de la classe, de se mettre à la place du parent, de s'assurer d'être clair dans les communications, de les inviter à venir à l'école, en leur expliquant ce qu'ils peuvent faire et en s'informant de leurs disponibilités (Hoover-Dempsey \& Sandler, 1997). Enfin, les enseignants identifient deux autres moyens pouvant favoriser les relations avec les parents, soit un au plan professionnel et un au plan personnel, soit améliorer la formation initiale et continue des enseignants sur les relations école-famille ou décrocher de son travail et prendre du temps pour soi.

En ce qui concerne des stratégies spécifiques aux parents, les enseignants n'en ont identifié aucune.

\section{Discussion des résultats}

\section{Les perceptions communes et spécifiques sur les stratégies}

D'une manière générale, l'analyse des résultats montre que les enseignants et les parents ont une vision relativement commune ou en continuité des stratégies à adopter. Ce constat montre ainsi le potentiel de mise en place de relations parent-enseignant harmonieuses, ce qui vient contrebalancer le discours mettant en évidence les malentendus, les difficultés et les obstacles de ces relations (Dubet, 1997; Crozier, 2000).

Il apparaît toutefois que les enseignants ont identifié un plus grand nombre de stratégies que les parents. On peut se questionner à savoir si cela témoigne d'une meilleure compréhension des relations par les enseignants ou d'attentes plus élevées de leur part. Par ailleurs, les enseignants identifient davantage que les parents des stratégies visant l'apprentissage (les résultats), ce qui peut dénoter une volonté de collaborer, mais dans un but précis lié à leur rôle principal, soit l'instruction. De leur côté, sans négliger l'aspect des apprentissages, les parents souhaitent d'abord que l'enseignant établisse une relation harmonieuse avec leur enfant, ce qui cohérent avec la documentation scientifique sur les attentes des parents d'enfants qui fréquentent le préscolaire et le primaire (Deslandes \& Bertrand, 2004; Larivée, 2012).

Bien que les parents identifient moins de stratégies que les enseignants, ils sont les seuls à avoir fait référence au travail d'équipe pour l'atteinte d'objectifs communs, mettant ainsi en évidence la relation enseignant-parents. Ceci peut s'expliquer par tout le discours sur l'importance de la collaboration école-famille ainsi que par une grande volonté de travailler en collaboration avec les ensei- 
gnants ou l'école (Caspe, Lopez \& Wolos, 2007; Peña, 2000), ce qui est souvent dénoncé comme étant trop faible ou difficile en raisons de nombreux obstacles (Dubet, 1997; Henderson, Mapp, Johnson \& Davies, 2007), les enseignants tentant trop souvent de protéger leur territoire (Lightfoot, 2003). Mais, encore faut-il que les enseignants soient aussi favorables au travail en équipe avec les parents. Ceci est d'autant plus important qu'il a été montré que l'attitude des parents à l'égard de l'enseignant ou de l'école influence l'attitude et le comportement de l'enfant en classe d'une part, et que l'attitude positive du parent par rapport à l'école influence positivement les enseignants (MacDonald, 2008).

Lorsque l'on porte une attention sur les types de stratégies identifiées, il apparaît que les enseignants et les parents ont principalement ciblé celles qui concernent les attitudes et comportements prosociaux puis, secondairement, l'implication parentale. Ces réponses ne sont pas surprenantes et sont cohérentes avec le thème de la recherche qui suppose que des relations parent-enseignant harmonieuses favorisent l'implication parentale (Henderson et al., 2007; Larivée, 2012). Il s'agit principalement de stratégies de base (ex.: être respectueux, inviter les parents, etc.), comme l'ont identifié plusieurs chercheurs (Epstein, 2001; Hoover-Dempsey \& Sandler, 1997), que l'on pourrait aussi identifier pour établir des relations interpersonnelles harmonieuses dans la vie en général. En outre, l'on peut retenir que les parents et les enseignants perçoivent, d'entrée de jeu, leurs relations comme toute autre relation interpersonnelle. Cependant, étant donné le contexte dans lequel s'établissent leurs relations et les enjeux qui sont sous-jacents (rôles respectifs, enseignement, apprentissage, réussite scolaire, etc.), la mise en application des stratégies mentionnées differe d'une relation habituelle et demeure un défi important. Leurs relations sont d'autant plus complexes du fait qu'elles sont teintées par d'autres facteurs, notamment la qualité de l'implication parentale dans le suivi scolaire de l'enfant et les performances des élèves, celles-ci pouvant être satisfaisantes ou pas tant pour les parents que les enseignants.

Concernant spécifiquement les apprentissages des élèves, seuls les enseignants ont identifié des stratégies. De leur côté, les parents ont identifié des stratégies diversifiées (appartenant à toutes les catégories, à l'exception de «apprentissages de l'enfant/élève»). Ceci montre, comme mentionné antérieurement, que les parents accordent plus d'importance à l'aspect relationnel qu'aux apprentissages, ces derniers étant favorisés par des relations de qualité.

Par ailleurs, les stratégies qui concernent spécifiquement les parents sont les moins nombreuses, et ce, tant de la part des enseignants que des parents. Ceci peut possiblement s'expliquer par le fait que l'enseignant détient une plus grande responsabilité dans l'établissement de la relation parent-enseignant. En effet, c'est habituellement l'école ou l'enseignant qui assure le leadership dans les relations interpersonnelles avec les parents (Hoover-Dempsey \& Sandler, 1997). Bien sûr, les parents peuvent solliciter des communications en présence ou à distance, mais, ultimement, c'est l'école qui décide d'y donner suite ou 
pas, rapidement ou non. Malgré les avancées des dernières années à l'égard de la collaboration école-famille, beaucoup de travail reste encore à faire, notamment au plan relationnel.

Au regard de ces résultats, il semble que l'établissement de relations parents-enseignants harmonieuses ne reposent pas sur des stratégies complexes ou très diversifiées. Les parents et les enseignants ont des perceptions similaires et ce sont plutôt les objectifs priorisés qui sont légèrement différents.

\section{Des Limites de La recherche}

Cette recherche a permis de mettre en évidence certaines stratégies relationnelles à l'égard de la collaboration école-famille. Cependant, ces résultats doivent être circonscrits à la présente recherche pour laquelle diverses limites s'imposent, ceci en raison de la taille et des caractéristiques de l'échantillon. En effet, le faible nombre de participants ainsi que leurs caractéristiques sociodémographiques (âge, statut socio-économique, région de résidence, niveau de scolarisation des parents, etc.) ne permettent pas une généralisation des résultats, ce qui n'était pas visé par la recherche. Plus particulièrement, mentionnons le statut socioéconomique des familles qui est assez élevé, ce qui ne permet pas de mettre en évidence des similitudes ou des différences entre les stratégies de familles aisées et défavorisées (Jacob \& Lefgren, 2007).

Un autre aspect qui témoigne des limites de la recherche concerne le contexte et les thèmes du protocole d'animation des groupes de discussions. En effet, nous sommes conscients que les réponses données par les participants à propos des questions traitant des stratégies relationnelles ont pu être influencées par les questions et réponses données aux autres thématiques abordées. Si nous avions questionné les participants seulement sur les questions relationnelles, possiblement que ceux-ci n'auraient pas abordé certains aspects vers lesquels nous les avons dirigés.

\section{Conclusion}

Les stratégies identifiées sont relativement en continuité et n'apparaissent pas «incompatibles» ou «irréconciliables». Les perceptions spécifiques sont plutôt des aspects différents identifiés par les enseignants et les parents que des aspects contraires. Les stratégies devraient cependant faire l'objet d'une plus grande explicitation et transparence (perceptions et attentes). Les enseignants et les parents doivent garder en tête qu'ils forment des entités hétérogènes (Crozier, 2000) même s'ils poursuivent un but commun, à savoir la réussite scolaire des enfants. À ce titre, l'établissement d'un dialogue sur les relations enseignant-parent pourrait permettre une plus grande harmonisation des attentes et perceptions respectives des parents et des enseignants à l'égard des stratégies éducatives à privilégier, ce qui s'avérerait bénéfique à tous y compris aux élèves. 
Enfin, bien que le discours général et la pression sociale prônent des relations parent-enseignant basées sur la collaboration et l'implication parentale, le constat à l'effet que seuls les parents parlent de travail en équipe pour l'atteinte d'objectifs communs soulève des questions sur les stratégies des enseignants, mais surtout sur leur conception de leur relation avec les parents. La crainte d'un partage du pouvoir ou de percevoir les parents comme des ennemis potentiels en serait-il une explication (Coleman, 1998; Lightfoot, 2003)? Si cela s'avérait fondé, il serait dès lors possible d'imaginer un obstacle significatif dans les relations. Il y a certes là des pistes de travail pour la poursuite de travaux de recherche.

\section{Note}

1 Par relations harmonieuses, nous référons à des relations entre parents et enseignants qui sont basées sur le respect mutuel, notamment de leurs rôles respectifs, et ce, au bénéfice de l'enfant/élève. Ces relations sont souvent qualifiées de «bonnes relations» par des enseignants et des parents.

\section{Références bibliographiques}

Bardin, L. (2013). L'analyse de contenu. Paris: PUF.

Beauregard, F. (2006). Représentations sociales des parents et des enseignants de leurs rôles dans l'intégration scolaire d'un élève dysphasique en classe ordinaire au primaire. Revue des sciences de l'éducation, 32(3), 545-565.

Brougère, G. \& Rayna, S. (2005). Accueillir et éduquer la petite enfance. Les relations entre parents et professionnels. Paris: INRP.

Caspe, M., Lopez, E. M. \& Wolos, C. (2007). Family involvement in elementary school children's education (Family Involvement Makes a Difference Research Brief No. 2, Winter 2006/2007). Cambridge, MA: Harvard Family Research Project.

Claes, M. \& Comeau, J. (1996). L'école et la famille: deux mondes? Lien social et politiques, $35,75-85$.

Coleman, P. (1998). Parent, student and teacher: The power of three. Thousand Oaks, Calif.: Corwin Press.

Crozier, G. (2000). Parents and schools. Partners or protagonists? Staffordshire: Trentham Books.

Deniger, M.-A., Anne, A., Dubé, S. \& Goulet, S. (2009). Les représentations du système scolaire des familles issues de milieux défavorisés. Montréal : Groupe de recherche sur l'éducation en milieu défavorisé du département d'administration et fondements de l'éducation de la Faculté des sciences de l'éducation, Université de Montréal.

Deslandes, R. \& Bertrand, R. (2004). Motivation des parents à participer au suivi scolaire de leur enfant au primaire. Revue des sciences de l'Éducation, 30, (2), 411-433.

Dom, L. \& Verhoeven, J. C. (2006). Partnership and conflict between parents and schools: How are schools reacting to the new participation law in Flanders (Belgium)? Journal of Education Policy, 21, (5), 567-597.

Dubet, F. (1997) (Éd.). École, familles: le malentendu. Paris: Textuel.

Epstein, J. L. \& Sanders, M. G. (2000). Connecting home, school, and community. In M. T. Hallinan (Ed.), Handbook of the sociology of education (pp. 285-306). New York, NY: Kluwer.

Epstein, J.L. (2001). School, family, and community partnerships: Preparing educators and improving schools. Boulder, CO: Westview Press.

Epstein, J.L. (2008). School, family, and community partnerships: Preparing educators and improving schools. Your handbook for action ( $3^{\mathrm{e}} \mathrm{ed}$.). Thousand Oaks, CA: Corwin Press. 
Garcia, D.C. (2004). Exploring connections between the construct of teacher efficacy and family involvement practices. Implications for urban teacher preparation. Urban Education, 39, (3), 290-315.

Gouvernement du Québec (2001). La formation à l'enseignement. Les orientations, les compétences professionnelles. Québec: Ministère de l'Éducation du Québec, Direction de la formation générale des jeunes.

Henderson, A. T. \& Mapp, K. (2002). A new wave of evidence: The impact of school, family, and community connections on student achievement. Austin, TX: Southwest Educational Development Lab.

Henderson, A. T., Mapp, K. L., Johnson, V. R. \& Davies, D. (2007). Beyond the bake sale. The essential guide to family-school partnerships. New York, NY: The New Press.

Hoover-Dempsey, K.V. \& Sandler, H.M. (1997). Why do parents become involved in their children's education? Review of Educational Research, 67, (1), 3-42.

Jacob, B. A. \& Lefgren, L. (2007). Low-income schools, parents want teachers who teach, Education Next (summer), 59-64.

Jeynes, W. H. (2011). Parental involvement and academic success. New York, NY: Routledge.

Larivée, S. J. (2011). L'établissement de relations école-famille collaboratives et harmonieuses. Des types d'activités, des obstacles, des enjeux et des défis. In J. Portelance, C. Borges \& J. Pharand (Éd.), La collaboration dans le milieu de l'éducation: dimensions pratiques et perspectives théoriques (pp. 161-180). Québec: Presses de l'Université du Québec.

Larivée, S. J. (2012). L'implication des parents dans le cheminement scolaire de leur enfant. Comment la favoriser? Éducation \& Formation, e-297, 33-48.

Larivée, S. J. (2010-2012). Les rôles éducatifs des parents et des enseignants et la participation parentale dans le cheminement scolaire de leur enfant au préscolaire ou au primaire: élaboration et validation d'un modèle théorique. Québec: Subvention de recherche du Conseil de recherche en sciences humaines du Canada (CRSHC), Initiatives de développement de la recherche (IDR).

Larivée, S. J. \& Larose, F. (2014). Les programmes d'implication parentale efficaces en milieux défavorisés: une recension des écrits. Revue internationale de l'éducation familiale, 36, 35-60.

Letarte, M. J., Nadeau, M. F., Lessard, J., Normandeau, S. \& Allard, J. (2011). Le rôle de la collaboration famille-école dans la réussite scolaire d'enfants ayant un déficit d'attention hyperactivité. Service social, 57, 20-36.

Lightfoot, S. L. (2003). The essential conversation: What parents and teachers can learn from each other. New York, NY: Random House LLC.

MacDonald, J. B. (2008). Teachers and parenting. In G. Olsen \& M. L. Fuller (Éd.), Home-school relations. Working successfully with parents and families (pp. 86-104). New York, NY: Pearson Education inc.

Ouédraogo, F. (2016). Étude des liens corrélationnels entre la collaboration école-famille, l'implication parentale, les styles éducatifs des parents et la réussite scolaire des élèves du primaire au Burkina Faso. Thèse de doctorat en Psychopédagogie et andragogie, Université de Montréal, Montréal.

Peña, D. C. (2000). Parent involvement: Influencing factors and implications. Journal of Educational Research, 94, (1), 42-54.

Périer, P. (2005). École et familles populaires: sociologie d'un différend. Rennes: Presses universitaires de Rennes.

Rayna, S., Rubio M.N. \& Scheu, H. (2010). Parents-professionnels: la coéducation en questions. Toulouse: Eres.

Reason, P. (1994). Three approaches to participative inquiry. In N.K. Denzin \& Y.S. Lincoln (Éd.), Handbook of Qualitative Research (pp. 324-339). Thousand Oakes, CA: Sage.

Rey, A., Robert, P., Rey-Debove, J. \& Cottez, H. (2006). Le Petit Robert de la langue française. Paris: Dictionnaire Le Robert. 
Mots-clés: Collaboration, relations, stratégies, enseignants, parents,

\section{Die Zusammenarbeit von Lehrpersonen und Eltern: Welche Strategien fördern eine harmonische Beziehung?}

\section{Zusammenfassung}

Seit den letzten vierzig Jahren wurde viel Forschung in Bezug auf die Beziehung von Schule und Familie geleistet. Diese konzentrieren sich auf die Zusasmmenarbeit mit den Eltern oder die Auswirkungen auf diese. Kaum wird auf die Beziehungsdynamik zwischen Eltern und Lehrpersonen fokussiert. In diesem Beitrag werden die Ergebnisse einer Forschung vorgestellt, die zum Ziel hat, Strategien zur Förderung für eine gelingende Beziehung zwischen Eltern und Lehrpersonen zu etablieren. Sie wurde mit Eltern $(\mathrm{N}=18)$ und Lehrpersonen $(\mathrm{N}=18)$ der Vorschule und der Primarstufe (Zyklus 1 bis 3, also 6- bis 11-Jährige) durchgeführt.

Wir stellen zunächst den Kontext und die Herausforderungen für die Beziehung zwischen Familie und Schule dar. Anschliessend werden die Strategien zur Förderung gelingender Beziehung vorgestellt. Allgemeine sowie spezifische Wahrnehmungen, die diese charakterisieren, werden identifiziert.

Schlagworte: Zusammenarbeit, Beziehung Familie-Schule, Strategien, gelingende Beziehung

\section{La collaborazione insegnanti-genitori: quali strategie per favorire relazioni armoniose? Riassunto}

\section{Riassunto}

Negli ultimi quarant'anni, sono state realizzate numerose ricerche sulle relazioni scuola-famiglia, ma esse s'interessano soprattutto alla collaborazione o all'implicazione dei genitori senza approfondire necessariamente la dinamica relazionale tra genitori e insegnanti. In questo testo presentiamo i risultati di una ricerca sulle strategie relazionali consone a favorire relazioni armoniose tra genitori e insegnanti. La ricerca è stata realizzata con i genitori di bambini $(n=18)$ e gli insegnanti $(\mathrm{n}=18)$ del livello prescolare (scuola materna, 5 anni) o elementare $\left(1^{\circ}, 2^{\circ}\right.$ e $3^{\circ}$ ciclo, ovvero da 6 a 11 anni). Presentiamo innanzitutto il contesto e gli ostacoli che circondano le relazioni scuola-famiglia. Esponiamo in seguito le strategie che favoriscono delle relazioni genitore-insegnante armoniose per poi identificare le percezioni comuni e specifiche che le caratterizzano.

Parole chiave: Collaborazione, relazioni, strategie, insegnanti, genitori 


\section{Teacher-Parent Collaboration: What Strategies for Fostering Harmonious Relationships?}

\section{Summary}

Over the last forty years, much research has been carried out on school-family relationships, but they have primarily focused on the collaboration or the involvement of parents without necessarily dealing in depth with the relational dynamics between parents and teachers. In this paper, we present the results of a research conducted with parents $(n=18)$ and preschool (kindergarten 5 years old) or primary school (1st, 2nd and 3rd cycle, 6 to 11 years old) teachers $(\mathrm{n}=18)$. The research focuses on relational strategies to promote harmonious relations between parents and teachers. We first present the context and obstacles to school-family relations. We then outline the strategies that promote harmonious parent-teacher relationships and we identify the common and specific perceptions that emerge.

Keywords: Collaboration, relationships, strategies, teachers, parents 
